\title{
GESTÃO DOS PARÂMETROS DE ESTOQUE: ESTUDO DE CASO DE ITENS DE MEDICAMENTOS EM FARMÁCIAS HOSPITALARES E CONVENCIONAIS
}

\section{INVENTORY STOCK PARAMETERS: CASE HISTORY FOR DRUG ITENS IN HOSPITAL AND CONVENTIONAL DRUGSTORES}

\author{
Letiene de Cássia Garcia ${ }^{1}$; Moacir Pereira ${ }^{2}$; Wislei Riuper Osório ${ }^{3}$ \\ ${ }^{1}$ Faculdade Network, Campus Nova Odessa, SP, Brasil \\ letienegarcia@yahoo.com.br \\ ${ }^{2}$ Centro Universitário Salesiano de São Paulo, Campus Americana, SP, Brasil \\ pereira moacir@yahoo.com.br \\ ${ }^{3}$ Faculdade Network, Campus Nova Odessa, SP, Brasil e Universidade de Campinas, UNICAMP - \\ Campinas,SP \\ wislei@fem.unicamp.br
}

\begin{abstract}
Resumo
Atualmente as farmácias têm utilizado uma politica de reposição de estoques para itens de medicamentos de baixo giro que tende a abarrotar ou repor em excesso os itens a serem repostos. A utilização de estoques, seja de segurança ou de cobertura para atender à demanda média durante o lead time é extremamente importante porque possibilita um melhor nível de atendimento ao cliente e melhora a competitividade da empresa em relação aos concorrentes. Neste contexto, o objetivo deste artigo é apresentar novos parâmetros de reposição estoques de medicamentos de baixo giro, especificamente os injetáveis, com base na filosofia de LEC (Lote Econômico de Compra). Para tanto, estudou-se um grupo de 10 itens de medicamentos da família dos injetáveis que são considerados de baixo giro. Com os novos parâmetros verificou-se uma considerável redução da quantidade desses 10 itens estudados e dos valores em estoques parados. Atingiram-se reduções em torno de $R \$ 750$ e $R \$ 90$ mil para as estudadas farmácias hospitalares e convencionais, respectivamente. Com a metodologia adotada para cálculo desses novos parâmetros de estoque, os níveis de serviço para atender aos clientes e ao balanceamento dos estoques foram mantidos.
\end{abstract}

Palavras-chave: controle de estoque; lote econômico de compra; ponto de pedido; estoques de segurança.

\section{Introdução}

O desafio do gestor de estoques é saber quando e quanto ressuprir de cada material e quanto deve manter em estoque de segurança. Com o crescente número de itens com diferentes padrões de demanda e características específicas, a complexidade na administração de materiais aumenta 
devido à necessidade de um controle diferenciado (SANTOS; RODRIGUES, 2006). A utilização de estoques, seja de segurança ou de cobertura, para atender à demanda média durante o lead time, é extremamente importante porque possibilita um melhor nível de atendimento ao cliente e melhora a competitividade da empresa em relação aos concorrentes.

A logística tem como foco principal a minimização do custo da operação para determinado nível de serviço e é um importante processo na viabilização da consecução do fluxo de suprimento de materiais no espaço e no tempo (BALLOU, 2001). A farmácia hospitalar depende de uma logística consistente e bem implementada quanto ao abastecimento de medicamentos, na medida em que lhe cabe prestar serviços de saúde a quem a procura, razão por que necessita ter em estoque medicamentos adequados ao receituário prescrito.

Atualmente estima-se que as farmácias têm utilizado modelos de reposição de estoque de medicamentos que tendem a abarrotar ou repor em excesso cada item do estoque. Espera-se que, com as sugestões de modificação do modelo de reposição de estoque, os proprietários das farmácias tenham ganhos significativos e a rede logística de abastecimento de medicamentos possa ser melhorada. O objetivo deste artigo é propor novos parâmetros de estoque, sendo eles: estoque de segurança, lote de compra, ponto de pedido, estoque máximo e tempos de ressuprimento com intuito de redução de custos e adequação dos estoques de medicamentos em farmácias de hospitalares e farmácias convencionais.

\section{Política de Gestão de Estoques}

A administração de materiais tecnicamente bem aparelhada é, sem dúvida, uma das condições fundamentais para o equilíbrio econômico e financeiro de uma empresa (FRANCISCHINI, 2002). A gestão de estoques é considerada como elemento fundamental para a redução e o controle dos custos totais e melhoria do nível de serviço prestado pelas empresas (WANKE, 2003, p. 11). A gestão de estoques constitui ainda uma série de ações que permitem ao administrador verificar se os estoques estão sendo bem utilizados, bem manuseados e bem controlados (DIAS, 1997). Em meados dos anos 80 houve uma "corrida" das empresas para baixarem seus estoques a todo custo, sem preocupação com os possíveis impactos correlacionados (CORREA et al., 2001).

Reporta-se em literatura (MARTINS; ALT, 2003; DIAS, 1997) dois aspectos importantes, isto é, o conhecimento da demanda e a obtenção dos materiais, podem influenciar decisivamente o estoque. Em literatura (KLASSEN; MENOR, 2007; SANDRINI; MESQUITA, 2008; KUNIGAMI; OSÓRIO, 2009) também se reporta que maiores níveis de capacidade de produção exigem menores níveis de estoque, e vice-versa, o que caracteriza um trade-off. Sandrini e Mesquita (2008) em 
estudos experimentais verificaram que o aumento da variação de demanda exige um maior estoque de segurança para acomodação ou minimização das incertezas. Verificou-se que o nível de serviço de $99,9 \%$ não foi conseguido nos casos em que o nível de utilização planejado é de $100 \%$, ainda que o nível de estoque seja muito alto.

Reporta-se que o controle de estoque é estudado desde 1913 com Harris ao introduzir a fórmula do lote econômico de compra (LENARD; ROY, 1995; KUNIGAMI; OSÓRIO, 2009). Para uma organização, a otimização do fluxo de materiais é de vital importância, pois os estoques representam grande parte dos seus custos logísticos. A gestão de estoques constitui uma série de ações que permitem ao administrador verificar se os estoques estão sendo bem utilizados, bem manuseados e bem controlados. Para Martins e Alt (2003), uma análise detalhada dos estoques é uma exigência pertinente a todo administrador de materiais. Isso se dá não somente em decorrência do volume de capital investido em estoque, mas, também, pela vantagem competitiva que a organização pode obter, dispondo de maior rapidez e precisão no atendimento ao cliente.

Na gestão dos estoques, os níveis de cada item e do próprio estoque como um todo devem ser revistos e atualizados periódica e constantemente para evitar problemas provocados em razão de maior demanda ou de sua redução, e alterações nos tempos de reposição. O conhecimento da demanda e a obtenção dos materiais, podem influenciar decisivamente o estoque (MARTINS; ALT, 2003). Para o caso da gestão hospitalar o estoque deve garantir a disponibilidade de medicamentos e materiais no momento e lugar necessários (DANIEL, 1997; PEREIRA, 2008). A adoção de certas regras define a forma de aquisição de materiais, procurando responder às questões de quando comprar e quanto comprar (TUBINO, 2004). Para tanto, a empresa deve definir e implantar alguns parâmetros de ressuprimento de estoques como: Estoque máximo ( $\mathrm{E}_{\text {máx. }}$ ) e Estoque de segurança $\left(E_{S}\right)$ que definem a quantidade de abastecimento, calculada com o estoque real acrescido das quantidades de encomendas aos fornecedores e a quantidade mínima possível capaz de suportar o tempo necessário para reabastecimento, respectivamente (TUBINO, 2004). Embora, existam autores que afirmem que, em muitas empresas, os estoques de segurança estão diretamente relacionados ao desvio padrão da previsão e que alguns combinem diferentes modelos de previsão com obtenção de melhores resultados (SANTOS; RODRIGUES, 2006), os valores de $E_{S}$ podem calculados a partir da Equação 1.

$E_{S}=f_{S} \times \sigma$

onde:

$\mathrm{f}_{\mathrm{s}}=$ fator de segurança (definido como função do nível de serviço desejado (TUBINO, 2004)); e $\sigma=$ desvio padrão das demandas avaliadas. 
Para a definição do ponto de pedido (PP) ou ressuprimento, o estoque de segurança deve ser somado com a demanda durante o lead time, independente da fórmula de cálculo (MARTINS; ALT, 2003; KREVER et al., 2003; TUBINO, 2004; BALLOU, 2001; PEREIRA, 2008; KUNIGAMI; OSÓRIO, 2009) como se segue:

$P P=\left(d_{t} \times t_{r}\right)+E_{S}$

onde:

$\mathrm{d}_{\mathrm{t}}=$ demanda diária; e

$\mathrm{t}_{\mathrm{r}}=$ tempo de reposição ou ressuprimento.

Para adoção do PP é importante que a quantidade a ser pedida seja definada, por meio do lote econômico de compras (LEC ou Q*), definido pela Equação 3:

$$
Q^{*}=\sqrt{\frac{2 D}{C} \cdot k}
$$

onde:

$\mathrm{D}=$ demanda no período;

$\mathrm{C}=$ custo unitário do medicamento no período;

$k=$ fator de poder de compra (PEREIRA, 2008; KUNIGAMI; OSÓRIO, 2009).

Críticas referentes aos modelos clássicos de estoque são comumente encontradas em literatura devido às limitações encontradas no confronto do modelo com o mundo real ou na determinação dos custos de pedido, de manutenção e encargos sobre o produto (ZOMERDIJK; VRIES, 2004; PEREIRA, 2008). Para isso aproximações podem ser feitas para minimizar esses efeitos.

\section{Metodologia}

Uma pesquisa entre farmácias hospitales e convencionais da RMC (Região Metropolitana de Campinas) foi realizada no intuito em se avaliar que tipo de política de reposição de medicamentos é utilizada. Na RMC estima-se que existem em torno de 50 hospitais, entre particulares, estaduais e municipais, dos quais 42 deles são particulares (84\%). A pesquisa só foi possível em três (03) unidades, sendo dois (02) da cidade de Americana e um (01) de Campinas. Os nomes dos respectivos hospitais são mantidos em anonimato por exigência dos mesmos. Torna-se importante 
ressaltar que a RMC tem abrangência de dezenove (19) municípios, com aproximadamente 2,5 milhões de habitantes.

Dentre as farmácias convencionais, cinco (05) cidades da RMC foram pesquisadas. Segundo informações obtidas no Sindicado das farmácias, o Sindfarma, da RMC, nessa região existem em torno de 4500 farmácias homologadas na cidade de Campinas e aproximadamente mais 3000 nas outras cidades integrantes da RMC. O tamanho da amostra pesquisada nesse tipo de farmácia é de 36 unidades, representando um percentual de apenas $0,5 \%$ do número total de farmácias integrantes da RMC. Dentre as 36 pesquisadas, quatro (04) diferentes redes de farmácias foram abordadas. Considera-se o percentual de $0,5 \%$ como bom nível de representatividade pois, daquelas pesquisadas, todas praticam similares filosofias de reposição e controle de estoques.

Sabe-se que o controle e gerenciamento de estoques multi-itens é bastante complexo devido à diversidade (PARTOVI; ANANDARAJAN, 2002). No entanto, para efeito de simplificação deste estudo, focou-se os itens chamados de injetáveis e de baixo giro para os quais avaliaram-se as demandas, quantidades mínimas e máxima de estocagem e também os lotes de segurança e de reposição (ou compra).

\section{Resultados e Discussões}

Embora a pesquisa tenha tido uma abrangência de um percentual de somente $10 \%$ das farmácias hospitalares e 0,5\% das convencionais na RMC, os gestores de estoques destas farmácias afirmaram que o atual sistema de abastecimento verificado é aplicado na maioria desses tipos de farmácias. Partindo-se desses dados, pode-se determinar uma equação que representa, em linhas gerais, o parâmetro que define os estoques máximo e mínimo, como função do consumo médio mensal (CMM). O mínimo é definido como o próprio CMM e o máximo sendo duas vezes o CMM expresso pela Equação 4. Em recente trabalho de doutoramento, Pereira (2008) também encontrou similar política de reposição de estoques para hospitais.

$$
C M M=\frac{\sum_{i=1}^{n}\left(\mathrm{Q}_{\mathrm{i}}\right)}{n}
$$

onde:

$\mathrm{Q}=$ quantidade consumida em cada período [mês];

$n=$ número de períodos; e

$i=$ índice do período. 
$\mathrm{Na}$ Tabela 1 exibem-se os custos unitários e as demandas nos períodos estudados para os 10 itens de medicamentos injetáveis, nas farmácias hospitalares e convencionais. Salienta-se que as farmácias hospitalares consideram 6 períodos (6 meses) para o cálculo de CMM, e as convencionais, somente 3 meses. Esses períodos foram mantidos para o cálculo dos novos parâmetros de estoque.

O sistema de controle de estoque de medicamento da farmácia irá sinalizar a necessidade de iniciar uma nova compra e respectiva reposição dos medicamentos, quando o nível de estoque de cada medicamento ficar igual ou menor que o valor exposto aos valores de PP. Importa esclarecer que o fator de segurança utilizado, neste caso, na composição da fórmula do Estoque de Segurança $\left(\mathrm{E}_{\mathrm{s}}\right)$ é igual a um (1), porque os medicamentos são considerados itens de "baixo giro" (nível de serviço próximo a 85\%) (TUBINO, 2004; BALLOU, 2001). O lote de compra econômico (Q*) calculado conforme Equação 3 considera um fator $k$ que é dependente tanto dos custos de preparação do pedido (A) quanto das taxas de encargos financeiros e armazenagem (I).

Tabela 1 - Descrição dos medicamentos pesquisados, valores de custo unitário conforme CMED* e as demandas em períodos mensais.

\begin{tabular}{|c|c|c|c|c|c|c|c|c|c|c|c|}
\hline \multirow{2}{*}{$\mathbf{N}^{\circ}$} & \multirow{2}{*}{$\begin{array}{l}\text { Medicamento } \\
\text { (descrição) }\end{array}$} & \multirow{2}{*}{$\mathbf{R} \$$} & \multicolumn{6}{|c|}{ Hospitalar } & \multicolumn{3}{|c|}{ Convencional } \\
\hline & & & P1 & $\mathbf{P 2}$ & $\mathbf{P 3}$ & P4 & P5 & P6 & P1 & $\mathbf{P 2}$ & P3 \\
\hline 1 & $\begin{array}{c}\text { Albumina humana } \\
20 \% \text { inj. }\end{array}$ & 67,58 & 7 & 8 & 4 & 4 & 6 & 7 & 0 & 1 & 1 \\
\hline 2 & Clexane 40 mg inj. & 417,03 & 130 & 115 & 191 & 153 & 147 & 139 & 41 & 35 & 33 \\
\hline 3 & Coltrax inj. & 9,36 & 179 & 195 & 156 & 114 & 168 & 179 & 215 & 233 & 199 \\
\hline 4 & Eprex $10.000 \mathrm{UI}$ inj. & $1.975,89$ & 95 & 99 & 119 & 99 & 112 & 98 & 5 & 3 & 2 \\
\hline 5 & Herceptin $440 \mathrm{mg}$ & $11.132,97$ & 3 & 6 & 5 & 3 & 4 & 2 & 0 & 1 & 0 \\
\hline 6 & Maxcef $2 \mathrm{~g}$ inj. & 113,44 & 665 & 566 & 439 & 534 & 572 & 529 & 206 & 211 & 208 \\
\hline 7 & Pantozol $40 \mathrm{mg}$ inj. & 159,12 & 28 & 33 & 37 & 31 & 28 & 32 & 8 & 7 & 5 \\
\hline 8 & $\begin{array}{l}\text { Tienam monovial } \\
\text { 500mg inj. }\end{array}$ & 114,72 & 54 & 98 & 182 & 154 & 122 & 139 & 5 & 2 & 5 \\
\hline 9 & Zoltec IV $100 \mathrm{ml}$ inj. & $1.221,00$ & 3 & 3 & 5 & 4 & 2 & 6 & 1 & 0 & 1 \\
\hline 10 & $\begin{array}{l}\text { Zometa } 4 \text { mg } 1 \mathrm{fr}+1 \\
\text { ampola }\end{array}$ & $1.424,16$ & 67 & 48 & 39 & 20 & 54 & 39 & 2 & 5 & 3 \\
\hline
\end{tabular}

(*) CMED - Câmara de Regulação do Mercado de Medicamentos da Agência Nacional de Vigilância Sanitária (ANVISA; 2008).

Para simplificação, neste artigo utilizaram-se dois valores desse fator, sendo: $k=50$ e $k=200$. O maior valor desse fator representa um aumento no poder de compra, ou de lote de reposição. Valores similares para esses fatores foram encontrados recentemente (PEREIRA, 2008).

Como exemplo, na Tabela 2 são indicados os valores de estoque de segurança $\left(\mathrm{E}_{\mathrm{s}}\right)$, Estoques máximos $\left(\mathrm{E}_{\text {máx. }}\right)$, ponto de pedido $(\mathrm{PP})$ e quantidade econômica de reposição $\left(\mathrm{Q}^{*}\right)$ para ambas as farmácias com fator $k=200$. 
Na Figura 1, tem-se uma comparação entre os níveis de estoques máximos e mínimos para o item $03\left(\right.$ Coltrax $\left.^{\circledR}\right)$ com fator de compra $(\mathrm{k}=50)$ pelo método CMM (atualmente praticado) e pela LEC (proposto) em farmácia convencional.

Figura 1 - Comparação entre níveis de estoques máximos e mínimos para o medicamento Coltrax ${ }^{\circledR}$ (item 03) em farmácia convencional com a política atual (CMM) e proposta (LEC) utilizando-se de fatores $k=50$.

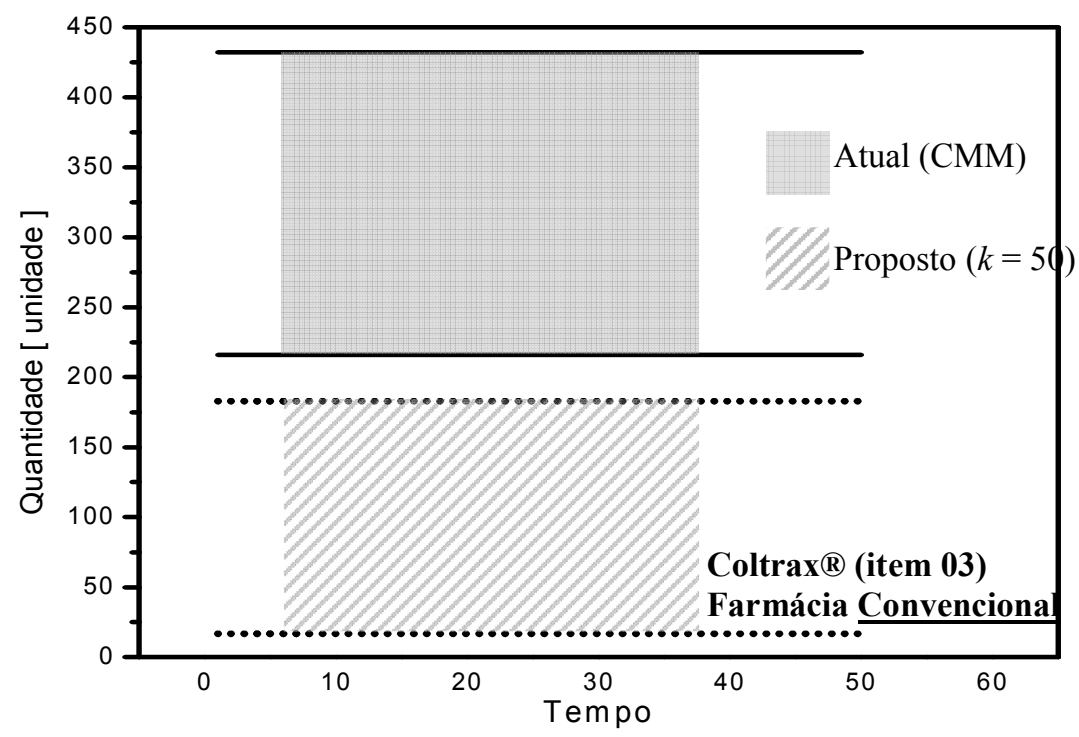

Fonte: Autoria Própria (2008)

Tabela 2 - Valores unitários de $\mathrm{E}_{\mathrm{s}}, \mathrm{E}_{\text {máx. }}, \mathrm{PP}, \mathrm{Q}^{*}$ e para farmácias hospitalar e convencional utilizando-se de fator de compra $k=200$.

\begin{tabular}{|ccccccccc|}
\hline \multirow{2}{*}{$\mathbf{N}^{\circ}$} & \multicolumn{4}{c}{ Farmácia HOSPITALAR } & \multicolumn{3}{c|}{ Farmácia CONVENCIONAL } \\
\cline { 2 - 9 } & $\mathbf{E}_{\mathbf{S}}$ & $\mathbf{E}_{\text {máx. }}$ & $\mathbf{P P}$ & $\mathbf{Q}^{*}$ & $\mathbf{E}_{\mathbf{S}}$ & $\mathbf{E}_{\text {máx. }}$ & $\mathbf{P P}$ & $\mathbf{Q}^{*}$ \\
\hline $\mathbf{1}$ & 2 & 22 & 3 & 21 & 1 & 7 & 1 & 7 \\
$\mathbf{2}$ & 26 & 67 & 50 & 41 & 4 & 25 & 10 & 20 \\
$\mathbf{3}$ & 28 & 319 & 56 & 291 & 17 & 350 & 53 & 333 \\
$\mathbf{4}$ & 10 & 25 & 27 & 16 & 2 & 4 & 2 & 3 \\
$\mathbf{5}$ & 1 & 3 & 2 & 1 & 1 & 1 & 1 & 0 \\
$\mathbf{6}$ & 73 & 226 & 165 & 153 & 3 & 96 & 37 & 94 \\
$\mathbf{7}$ & 3 & 34 & 9 & 31 & 2 & 16 & 3 & 14 \\
$\mathbf{8}$ & 45 & 117 & 66 & 72 & 2 & 15 & 2 & 13 \\
$\mathbf{9}$ & 1 & 5 & 2 & 4 & 1 & 2 & 1 & 2 \\
$\mathbf{1 0}$ & 16 & 28 & 23 & 12 & 2 & 5 & 2 & 3 \\
\hline
\end{tabular}

Fonte: Autoria Própria (2008)

Observa-se que com a determinação de novos parâmetros de estoque, conforme filosofia LEC, que os níveis de estoques são consideravelmente "deslocados" para menores níveis. Salientase que itens que apresentem PP maior que $\mathrm{E}_{\text {máx }}$ é um indicativo de que o lead-time de ressuprimento 
ou tempo de reposição utilizado para cálculos $\left(\mathrm{t}_{\mathrm{R}}=5\right.$ dias) deve ser reduzido. Assim, o item 4 (com $\mathrm{k}=50$ ) requer $u m t_{\mathrm{R}}=2$. Comparações entre os estoques máximos e mínimos (segurança) calculados pela atual política e a proposta por este artigo (fatores $\mathrm{k}=50$ e 200) são apresentadas na Fig.2(a) e (b) e Fig.3(a) (b), para farmácias hospitalar e convencional, respectivamente.

Figura 2 - Comparações entre estoques máximos para os 10 medicamentos estudados com a política atual de reposição e a proposta para: (a) farmácia hospitalar e (b) convencional com fatores $k=50$ e 200.

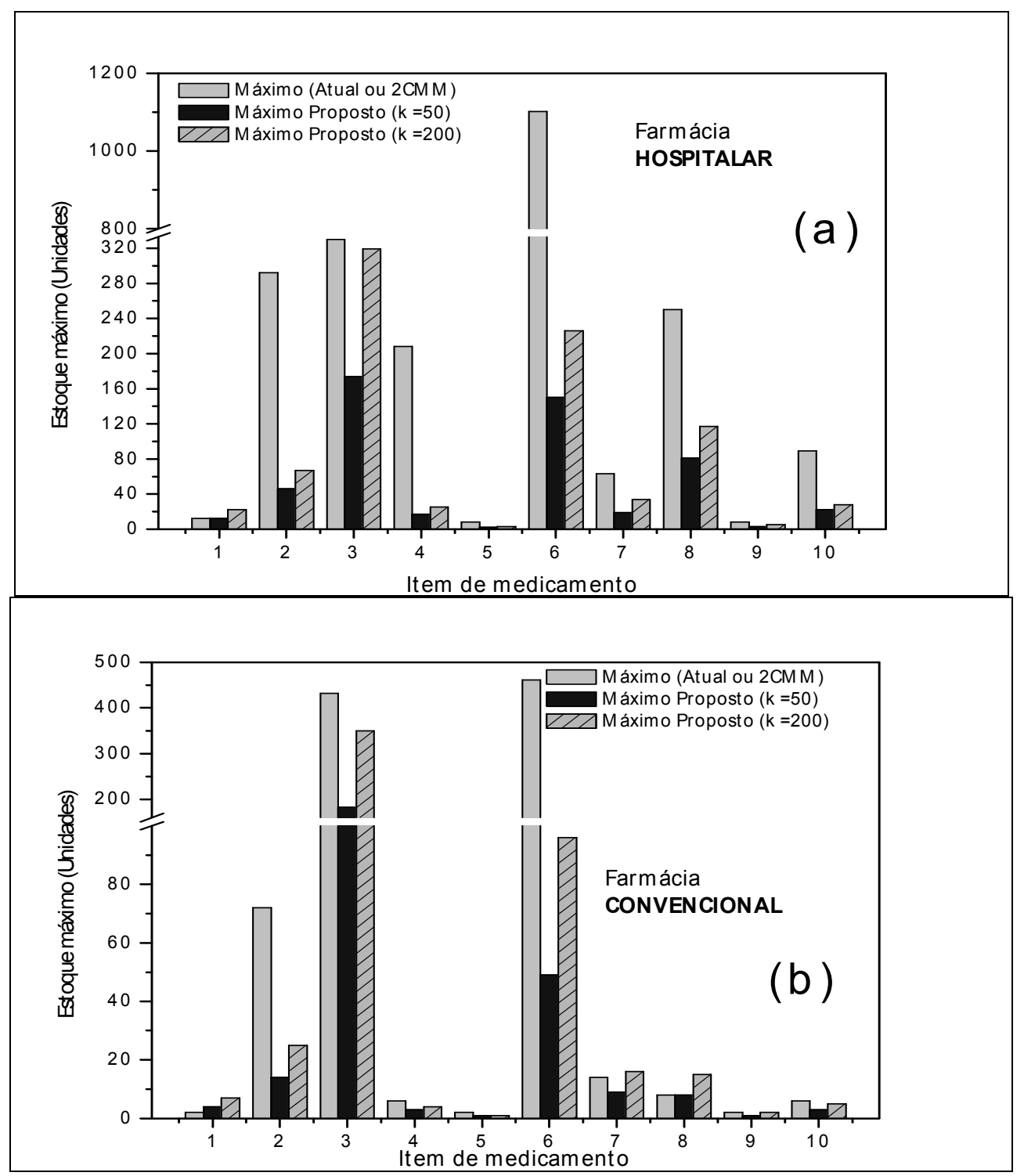

Fonte: Autoria Própria (2008)

A partir das comparações entre os níveis de estoque de medicamentos paras as farmácias hospitalar e convencional, tomando-se como base a atual política e a proposta no presente artigo, para os 10 itens pesquisados e com dois diferentes "poderes de compra" (fatores $k=50$ e $k=200$ ), permite-se concluir que a política proposta apresenta menores níveis de estocagem na maioria dos 
medicamentos estudados para os dois tipos de farmácias, que apresentam naturezas diferentes de atendimento e também diferenças significativas nas demandas dos medicamentos estudados.

Figura 3 - Comparações entre estoques de segurança $\left(E_{\mathrm{s}}\right)$ para os 10 medicamentos estudados com a política atual de reposição e a proposta para: (a) farmácia hospitalar e (b) convencional com fatores $k=50$ e 200.

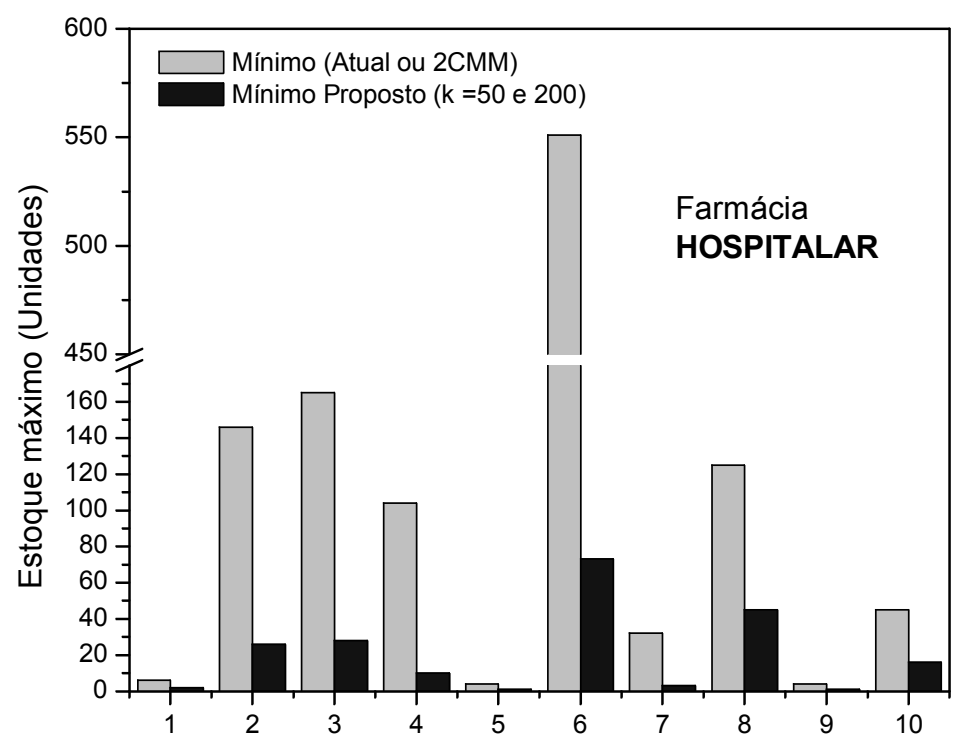

(a)

Item de medicamento

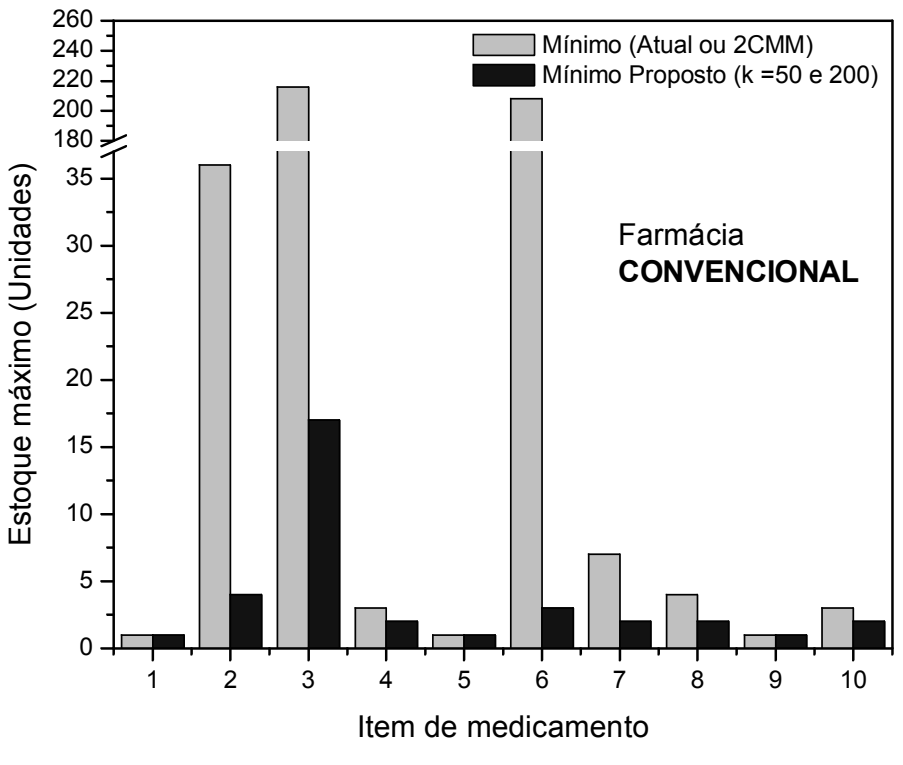

Fonte: Autoria Própria (2008)

Na Figura 2(a) e (b) observa-se que a proposta política de gestão de estoque pode não ser validada para o item 01, na farmácia hospitalar, e nos itens 01 e 08 para a farmácia convencional. Isso significa que os estoques máximos destes itens ficariam sutilmente elevados em relação à atual política (2CMM). Isso deve-se ao fato de que estes, além de representarem itens de baixo giro, são também classificados como itens da classe "C", com representatividades muito pequenas na 
avaliação de demanda valorizada, sendo de 0,09\% para o item 01 da farmácia hospitalar e de 0,08 e 0,81\%, respectivamente, para os itens 01 e 08 da farmácia convencional. Além disso, verifica-se que os itens 01, 05 e 09 são aqueles que podem ser considerados de "ultra-baixa" demanda, e por essa razão, as políticas (CMM) e por PP e LEC não apresentam consideráveis diferenças. Embora não apresentem correlações entre as demandas nos dois tipos de farmácias, os três itens $(01,05$ e 09$)$ apresentam classificações similares, ou seja, classe "B" para item 05 e classe "C" para os itens 01 e 09.

Em uma análise financeira comparativa dos volumes montantes ou níveis de medicamentos estocados pela atual política (CMM) e pela política proposta, verificam-se consideráveis diferenças. Na Figura 4 exibe-se uma representação em termos valorizados das possíveis diferenças de economias na gestão dos estoques.

Figura 4 - Comparações entre valores dos estoques em plena carga para os 10 medicamentos estudados com a política atual de reposição e a proposta para: (a) farmácia hospitalar e (b) convencional com fatores $k=50$ e 200.

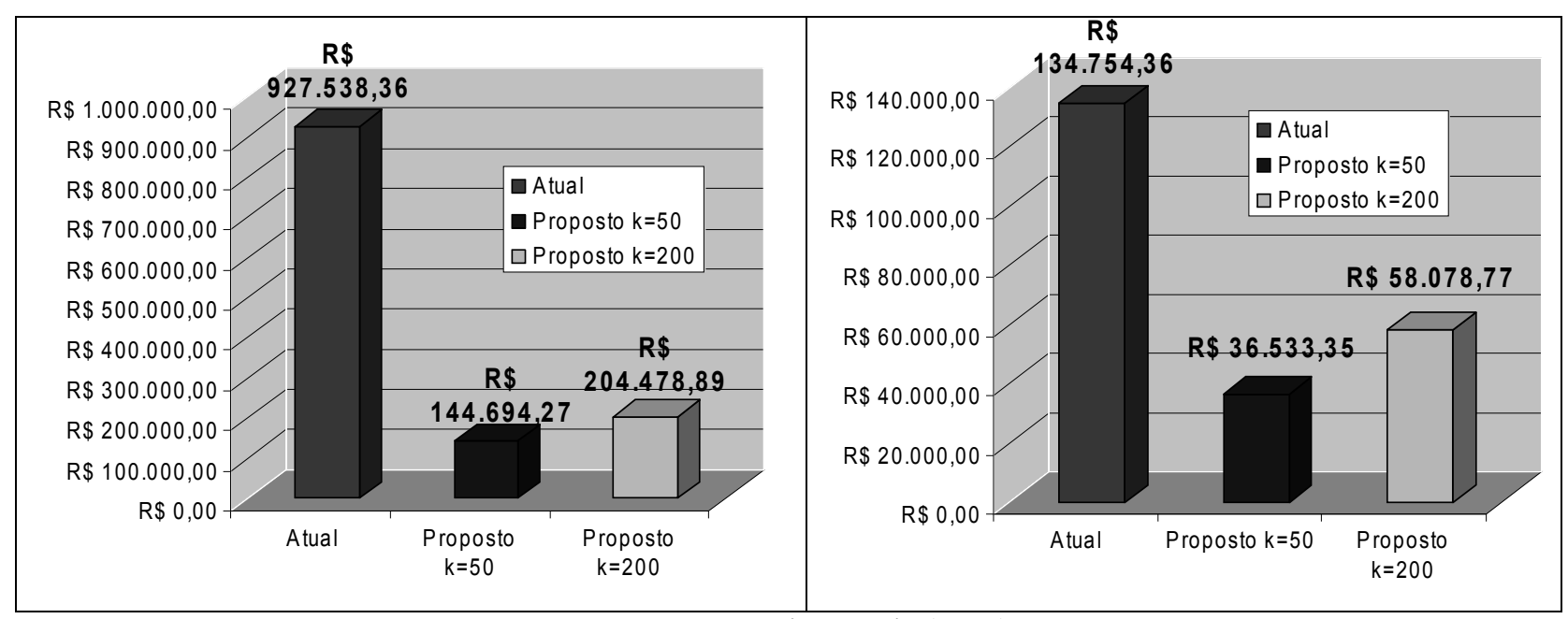

Fonte: Autoria Própria (2008)

No caso das farmácias hospitalares, pode-se atingir uma “economia" em torno de R\$750 mil para a carga plena dos estoques, uma redução para até 1/6 do valor gasto para a reposição plena dos estoques; isso levando-se em conta que seria necessário um maior espaço físico para o armazenamento de quase 2500 itens para a atual política, e somente por volta de 700 itens com a nova proposta. No caso da farmácia convencional estudada, a “economia” seria por volta de R\$90 mil, o que representaria uma redução para até $1 / 4$ do valor anterior, dependendo do poder de compra que se deseja atingir, como função dos valores de $k$. A quantidade de itens seria reduzida de mais de 1000 unidades para perto de 300 a 500 unidades com a política proposta. 


\section{Considerações Finais}

O intuito central deste artigo foi avaliar que tipo de política de gestão de abastecimento de medicamento estava sendo colocada em prática nas farmácias de hospitais particulares e farmácias convencionais da RMC. Para isso, universos amostrais consideráveis foram estudados, e a partir dos resultados obtidos, pôde-se concluir que a proposta política de ressuprimento de medicamentos com base no cálculo de LEC e PP (lote econômico de compra e ponto de pedido) traz consideráveis reduções, tanto nos volumes (quantidades) de itens a serem estocados, quanto nos valores do estoques "parados".

Além disso, observou-se que as farmácias hospitalares apresentam um lead-time de reposição de 5 dias. Do contrário, as farmácias hospitalares podem apresentar ciclos de reposição de até 1 dia. Isso pode significar "perdas" ou "custos" desnecessários para fomentar o sistema logístico que atende às farmácias, onde o transporte, o acondicionamento, o número de funcionários e o nível de satisfação dos clientes podem ser significativamente "abalados". Isso principalmente porque a maior parte dos itens não exige reposição diária, como têm praticado as farmácias convencionais. Neste artigo, o modelo de processo de reposição de medicamentos na farmácia dos hospitais pesquisados, com a finalidade de reduzir o tempo de abastecimento, em busca de adequação e equilíbrio no estoque de medicamentos mostrou-se satisfatório. Os resultados mais expressivos podem ser sintetizados em "ganhos" significativos ou "gastos desnecessários" com a atual política de gestão de estoques.

No caso das farmácias hospitalares, pode-se atingir uma “economia” em torno de R\$750 mil para a carga plena dos estoques, uma redução para até 1/6 do valor gasto para a reposição plena dos estoques. Isso se levar em conta que seria necessário um maior espaço físico para o armazenamento de quase 2500 itens para a atual política e somente por volta de 700 itens com a nova proposta.

No caso da farmácia convencional estudada, a "economia" seria por volta de R\$90 mil, o que representaria uma redução para até $1 / 4$ do valor inicial, dependendo do poder de compra que se deseja atingir, como função dos valores de $k$. A quantidade de itens seria reduzida de mais de 1000 unidades para perto de 300 a 500 unidades, com a política proposta.

Uma limitação verificada neste artigo, ou seja, da aplicação do modelo proposto de gestão (reposição) de estoques, consiste na similaridade dos resultados para itens considerados de demanda ultra-baixa. Além disso, cuidados devem ser tomados para medicamentos ou produtos que, por efeitos de cálculos da relação demanda e custo, podem afetar significativamente os lead-times de reposição, onde em determinadas situações esse tempo deve ser reduzido e em outras situações esse tempo pode ser ampliado. 


\title{
Agradecimentos
}

Os autores agradecem todo o apoio dado pelos colaboradores e gestores da HAB, Honda Automóveis Brasil, Sumaré e pelas informações fornecidas quando solicitadas. Agradecimentos também são direcionados ao aporte financeiro provindo das Faculdades Network, Sumaré, SP.

\begin{abstract}
Nowadays a high volume of stocked items has been attained due to a replacement method for drug items with low demand cycle is applied. Safety and replenishment stocks are intensively used to foment the mean demand during lead-time. This is extremely important in order to provide improvements at the customer attendance level and at competitiveness compared to its competitors. The aim of this article is focused to evaluate new parameters for replenishment stocks of the low demand cycle, particularly those injecting drug group, based on the replenishment economic lot (REL) method. A 10 drug items of low demand cycle into injecting drugs family was chosen. It was attained a considerable decreasing in the stocked volume of these 10 items and in the standby stock level. It was found decreasing of about $\mathrm{R} \$ 750,000.00$ and $\mathrm{R} \$ 90,000.00$ for hospital and conventional drugstores, respectively. Considering the proposed method for determine stock parameters, the customer's attendance levels and the stock balance have been provided.
\end{abstract}

Key-words: inventory stock control; replenishment economic lot; requering point, safety stock.

\section{Referências}

ANVISA - Agência Nacional de Vigilância Sanitária, Disponível em: http://www.anvisa.gov.br/monitora/precos.htm, Acesso em: 12 jan 2008.

BALLOU, R. H. Gerenciamento da cadeia de suprimentos. Planejamento, organização e logística empresarial. 4. ed. São Paulo: Bookman, 2001.

CHAN, C. K.; KINGSMAN, B. G.; WONG, H. The value of combining forecasts in inventory management: a case study in banking. European Journal of Operational Research, vol. 117, p. 199-210, 1999.

cross'

CORREA, H. L.; GIANESI, I. G. N; CAON, M. Planejamento, Programação e Controle da Produção. 4. ed. São Paulo, 2001

DANIEL, W. Applying Just-in-time Systems in Health Care. II E Solutions, v. 29, n. 8, p. 32, 1997.

FRANCISCHINI, Paulino G.; GURGEL, Floriano do Amaral. Administração de materiais e do Patrimônio. São Paulo: Pioneira, 2002.

DIAS, M. A. P. Administração de materiais: resumo da teoria, questões de revisão, exercícios, estudos de caso. Edição Compacta. $4^{a}$ ed. São Paulo: Atlas, 1997.

KLASSEN, R.; MENOR, L. The process management triangle: An empirical investigation of process trade-offs. Journal of Operations Management, v.25, p. 1015-1034, 2007.

crossef

KUNIGAMI, F.J; OSÓRIO, W.R. Gestão no controle de estoque: estudo de caso em montadora automobilística, Revista Gestão Industrial, submetido 2009.

KREVER, M.; WUNDERINK, S.; DEKKER, R.; SCHORR, B. Inventory control based on advanced probability theory, an application. European Journal of Operational Research, vol. 162, p. 342-358, 2003. 
LENARD, J. D.; ROY, B. Multi-item inventory control: A multicriteria view. European Journal of Operational Research, vol. 87, p. 685-692, 1995.

cross ${ }^{\text {ef }}$

MARTINS, P.G., ALT, P.R.C. Administração de Materiais e Recursos Patrimoniais. São Paulo, Saraiva, 2003.

PARTOVI, F. Y.; ANANDARAJAN, M. Classifying inventory using an artificial neural network approach. Computers and Industrial Engineering, vol. 41, p. 389-404, 2002.

cross'

PEREIRA, M., Logística Hospitalar: Reposição Contínua de Medicamentos, Moinho Editorial, ISBN: 856143700-6, $1^{\text {a }}$. edição, p. 128, 2008.

SANDRINI, L.G.; MESQUITA, M. A. O trade-off entre a folga na capacidade de produção e o nível de estoque, Revista Gestão Industrial, v. 04, n. 04: p. 217-233, 2008

SANTOS, A.M., RODRIGUES, I. A. Controle de Estoque de Materiais com Diferentes Padrões de Demanda: Estudo de Caso em uma Indústria Química, Gestão \& Produção, vol. 13, n.2, pp.223-231, 2006.

cross ref

TUBINO, D.F. Manual de Planejamento e Controle da Produção. São Paulo, Atlas, 2004.

ZOMERDIJK, L. G.; VRIES, J. An organizational perspective on inventory control: Theory and a case study. International Journal of Production Economics, vol. 81-82, p. 173-183, 2003.

crossef

WANKE, P. Gestão de Estoques na Cadeia de Suprimentos: Decisões e Modelos Quantitativos. Rio de Janeiro: Editora Atlas, 2003.

\section{Dados dos autores:}

Letiene Cássia Garcia

Faculdades Network

Administradora de Empresas

Endereço: Av. Ampélio Gazzetta, 2445, 13460-000, Nova Odessa, SP, Brazil

Telefones para contato: (55) 1935213319 ou 1991500988

e-mail: letienegarcia@yahoo.com.br

\section{Moacir Pereira}

Centro Universitário Salesiano de São Paulo, Campus M. Auxiliadora, Americana, SP

Prof. Dr. Engenharia de Produção

Professor do Curso de Mestrado da UNISAL, Americana

Endereço: Av. De Cillo, 3500, Parque Universitário, 13467-600, Americana, SP

Telefones para contato: (55) 193471 9760, Fax.: 551934719759

e-mail: pereira_moacir@yahoo.com.br

\section{Wislei Riuper Osório}

Faculdades Network e UNICAMP, Universidade Estadual de Campinas 
Administração e Engenharia de Materiais

Coordenador de Curso de Administração, Pesquisador Colaborador

Endereço: Av. Ampélio Gazzetta, 2445, 13460-000, Nova Odessa, SP, Brazil

Telefones para contato: (55) 1935213319 ou 1991500988

e-mail: wislei@,fem.unicamp.br ou adm@nwk.edu.br

Recebido para publicação em: 06/02/2009

Aceito para publicação em: 16/03/2009 